\title{
A Novel Murine Model of Parvovirus Associated Dilated Cardiomyopathy Induced by Immunization with VP1-Unique Region of Parvovirus B19
}

\author{
Julijus Bogomolovas, ${ }^{1,2}$ Egidijus Šimoliūnas, ${ }^{3}$ Ieva Rinkūnaitè, ${ }^{3}$ \\ Luka Smalinskaitè, ${ }^{1}$ Andrej Podkopajev, ${ }^{2}$ Daiva Bironaitè, ${ }^{4}$ \\ Cleo-Aron Weis, ${ }^{5}$ Alexander Marx, ${ }^{5}$ Virginija Bukelskienè, ${ }^{3}$ Norbert Gretz, ${ }^{6}$ \\ Virginija Grabauskienè, ${ }^{2}$ Dittmar Labeit, ${ }^{1}$ and Siegfried Labeit ${ }^{1}$ \\ ${ }^{1}$ Department of Integrative Pathophysiology, Medical Faculty Mannheim, Mannheim, Germany \\ ${ }^{2}$ Department of Pathology, Forensic Medicine and Pharmacology, Faculty of Medicine, Vilnius University, Vilnius, Lithuania \\ ${ }^{3}$ Department of Biological Models, Institute of Biochemistry, Vilnius University, Vilnius, Lithuania \\ ${ }^{4}$ State Research Institute, Centre for Innovative Medicine, Department of Stem Cell Biology, Vilnius, Lithuania \\ ${ }^{5}$ Medical Faculty Mannheim, Institute of Pathology, Mannheim, Germany \\ ${ }^{6}$ Medical Faculty Mannheim, Medical Research Centre, Mannheim, Germany \\ Correspondence should be addressed to Julijus Bogomolovas; julius.bogomolovas@medma.uni-heidelberg.de
}

Received 17 May 2016; Revised 9 August 2016; Accepted 14 September 2016

Academic Editor: Andrea Vecchione

Copyright ( 2016 Julijus Bogomolovas et al. This is an open access article distributed under the Creative Commons Attribution License, which permits unrestricted use, distribution, and reproduction in any medium, provided the original work is properly cited.

\begin{abstract}
Background. Parvovirus B19 (B19V) is a common finding in endomyocardial biopsy specimens from myocarditis and dilated cardiomyopathy patients. However, current understanding of how B19V is contributing to cardiac damage is rather limited due to the lack of appropriate mice models. In this work we demonstrate that immunization of BALB/c mice with the major immunogenic determinant of B19V located in the unique sequence of capsid protein VP1 (VP1u) is an adequate model to study B19V associated heart damage. Methods and Results. We immunized mice in the experimental group with recombinant VPlu; immunization with cardiac myosin derived peptide served as a positive reference and phosphate buffered saline served as negative control. Cardiac function and dimensions were followed echocardiographically 69 days after immunization. Progressive dilatation of left ventricle and decline of ejection fraction were observed in VPlu- and myosin-immunized mice. Histologically, severe cardiac fibrosis and accumulation of heart failure cells in lungs were observed 69 days after immunization. Transcriptomic profiling revealed ongoing cardiac remodeling and immune process in VPlu- and myosin-immunized mice. Conclusions. Immunization of BALB/c mice with VPlu induces dilated cardiomyopathy in BALB/c mice and it could be used as a model to study clinically relevant B19V associated cardiac damage.
\end{abstract}

\section{Introduction}

Dilated cardiomyopathy (DCM) remains the leading cause of heart failure leading to heart transplantation. When therapeutic and prophylactic measures are applied early, a progression of ischemic cardiomyopathy can be stopped and patient can be saved from the heart transplantation. However, only in selected cases progression of primary nonischemic dilated cardiomyopathy can be circumvented with the currently available therapy. Thus, a high demand for new DCM therapies exists. Improved diagnostics revealed that up to $70 \%$ of primary nonischemic DCM cases might be due to an unfortunate combination of immunological factors and viral agents [1].

Numerous reports on the presence of parvovirus B19 (B19V) in the hearts of DCM patients imply that B19V might be causatively linked to the heart damage, but the understanding of its pathogenicity in heart diseases is rather 
limited [2]. B19V infection in humans typically manifests as erythema infectiosum, hydrops fetalis in pregnant women or aplastic crisis, infecting and replicating in erythroid progenitor cells. Up to $60 \%$ of adult population are positive for B19V IgG antibodies indicating a previous infection [3]. Following the $\mathrm{B} 19 \mathrm{~V}$ infection, a dominant immune response is elicited by the VP1-unique region (VP1u), which harbors potent neutralizing epitopes $[4,5]$. VP1u is an N-terminal 227 amino acid long region distinguishing minor capsid component VP1 from the major VP2 protein. VP1u region possesses phospholipase activity critical for infectivity [6] and acts as a major determinant of viral tropism [7]. Upon cardiac B19V infection, viral genomes are detected only in endothelial cells and not in myocytes [2]. Therefore, it is very likely that mechanisms beyond the viral infection are involved in the pathogenesis of B19 related DCM. It has been proposed that viral infection can trigger pathological immune response leading to the development of DCM [1].

So far two studies reported on cardiac response to VPlu immunization. Active immunization of $B A L B / c$ mice with VPlu induced cardiac inflammation and increased blood levels of nonspecific cytosolic enzymes [8]. Passive immunization of ZB/W F1 mice with rabbit anti-B19-VP1u IgG elicited foci of cardiac infiltration and upregulation of cardiac matrix metalloproteinase-9 (MMP9) activity. However, no significant differences in the cardiac atrial natriuretic peptide, brain natriuretic peptide, heart-type fatty acid-binding protein, and creatine kinase MB levels were detected among all experimental groups [9]. Nevertheless, cardiac function was not evaluated in these studies and progression/resolution of cardiac damage was not followed in these studies. Thus, in this work we demonstrate that VPlu holds cardiopathic potential in the experimental paradigm of widely accepted myosininduced myocarditis model. Cardiac damage is induced by immunization of $\mathrm{BALB} / \mathrm{c}$ mice with a cardiac myosin derived peptide. Immunized animals developed acute myocarditis accompanied inflammatory infiltrates about 21 days after immunization and subsequent progression to DCM marked by cardiac fibrosis and impaired systolic function around 40 to 70 days after immunization [10]. To our knowledge, this is the first murine model to study B19V induced DCM.

\section{Materials and Methods}

2.1. Animal Experiments. Experiments were performed on 6-8-week-old BALB/c male mice. Animals were housed in standard plastic cages in an animal room with controlled environmental conditions and maintained on standard food and water ad libitum. Experimental procedures conformed to Directive 2010/63/EU requirements and were approved by the Lithuanian State Food and Veterinary Service (approval number G2-17, 2014/11/11).

2.2. Immunization Protocol. Mice were immunized as described [10]. A peptide derived from $\alpha$-myosin (MyHC- $\alpha 6$ 14-62 RSLKLMATLFSTYASADR) or recombinant VPlu (residues 3-229, UniProtKB/Swiss-Prot: P07299.1) produced in endotoxin-deficient ClearColi (Lucigen) E. coli strain as described earlier [11] was used for immunization.
$150 \mu \mathrm{g} /$ animal of cardiac myosin or $500 \mu \mathrm{g} /$ animal of VP1u was used per immunization. Animals were immunized twice in a 7-day interval. Peptide and protein dissolved in PBS were mixed with equal amount of Freund's complete adjuvant for the first and incomplete Freud's adjuvant for second immunization, emulsified using sonifier, and injected subcutaneously. During the first immunization, animals were stimulated with $500 \mathrm{ng}$ intraperitoneal injection of Pertussis toxin in bovine serum albumin supplemented sterile saline. Control animals were immunized using PBS mixed with Freund's adjuvant following the same protocol described above.

2.3. Echocardiography. Transthoracic M-mode echocardiography was performed under isoflurane (1\% in pure oxygen) anesthesia, using linear $13 \mathrm{MHz}$ sensor coupled to HITACHI EUB-7000HV ultrasound system. The heart was viewed in short parasternal axis at the papillary muscle level. Six cardiac cycles per measurement were used for averaging. Animals were examined on the day of the first immunization and then 20,47, and within 60-69 days later. LV ejection fraction was calculated using Teichholz method from M-mode images [12].

2.4. Histology. 60 to 69 days after immunization, animals were sacrificed and heart and lungs were fixed in $10 \%$ neutral buffered formalin, embedded in paraffin, sectioned at $6 \mu \mathrm{m}$, and stained with picrosirius or Prussian blue (ScyTek) following manufacturer's recommendations. The degree of fibrosis was graded by an experienced pathologist on picrosirius stained sections under white light; density of alveolar siderophages in lung parenchyma was quantified using semiautomated routine in Fiji [13]. For CD45 immunohistochemistry, reagents from Dako Ltd. were used. Heatmediated antigen retrieval was performed. Dakoreal ${ }^{\mathrm{TM}} \mathrm{EN}$ Vision TM Detection System Peroxidase/DAB was used to visualize the signal. Rabbit polyclonal anti-CD45 antibody (Abcam ab10558) was used at 1:1000 dilution. CD45 positive foci were quantified by counting in 10 random fields (magnification 400x).

2.5. Expression Profiling. Total RNA was extracted from frozen apical LV region using TRIzol ${ }^{\circledR}$ Plus RNA Purification Kit (ThermoFisher Scientific). Gene expression profiling with Affymetrix system using GeneChip ${ }^{\circledR}$ Mouse Gene 2.0 ST Arrays was performed in Microarray Core Facility at Medical Research Center. Arrays were processed using software package JMP ${ }^{\circledR}$ Genomics. Gene set enrichment analysis was performed using GSEA-P [14] with KEGG pathway database derived gene sets [15]. Gene sets with $p$ value and $q$-val FDR $<0.05$ were considered significantly dysregulated and visualized with Enrichment Map [16].

2.6. Statistics. Changes in echocardiographic parameters were statistically evaluated using mixed ANOVA. Differences between fibrosis scores and alveolar siderophage densities were statistically assessed using The Kruskal-Wallis $H$ test. The analysis was performed using SPSS 23; $p$ values $<0.05$ were considered significant. 
TABLE 1: Echocardiographic parameters of immunized animals.

\begin{tabular}{|c|c|c|c|c|c|}
\hline \multirow[t]{2}{*}{ Antigen } & \multirow{2}{*}{$\begin{array}{c}\text { Days after } \\
\text { immunization }\end{array}$} & $\begin{array}{l}\text { LV end-diastolic } \\
\text { diameter }(\mathrm{mm})\end{array}$ & $\begin{array}{l}\text { LV end-systolic } \\
\text { diameter (mm) }\end{array}$ & $\begin{array}{l}\text { Fractional shortening } \\
(\%)\end{array}$ & $\begin{array}{l}\text { LV ejection fraction } \\
(\%)\end{array}$ \\
\hline & & Mean \pm SE & Mean \pm SE & Mean \pm SE & Mean \pm SE \\
\hline \multirow{4}{*}{ PBS } & 0 & $3.93 \pm 0.10$ & $2.64 \pm 0.10$ & $32.9 \pm 1.3$ & $61.9 \pm 1.8$ \\
\hline & 20 & $4.12 \pm 0.14$ & $2.70 \pm 0.14$ & $34.7 \pm 1.5$ & $64.2 \pm 2.1$ \\
\hline & 47 & $4.05 \pm 0.10$ & $2.66 \pm 0.07$ & $34.4 \pm 1.3$ & $64.0 \pm 1.8$ \\
\hline & $60-69$ & $4.25 \pm 0.10$ & $2.83 \pm 0.19$ & $33.6 \pm 3.2$ & $62.3 \pm 4.2$ \\
\hline \multicolumn{2}{|c|}{$\begin{array}{l}\text { Simple main effect for } \\
\text { measurement time }\end{array}$} & $\begin{array}{c}F(3,9)=1.025, p= \\
0.426, \text { partial } \eta^{2}= \\
0.255\end{array}$ & $\begin{array}{c}F(3,9)=0.574, p= \\
0.646, \text { partial } \eta^{2}= \\
0.161\end{array}$ & $\begin{array}{c}F(3,9)=1.010, p= \\
0.432, \text { partial } \eta^{2}= \\
0.252\end{array}$ & $\begin{array}{c}F(3,9)=1.060, p= \\
0.413 \\
\text { partial } \eta^{2}=0.261\end{array}$ \\
\hline \multirow{4}{*}{$\begin{array}{l}\text { Cardiac } \\
\text { myosin }\end{array}$} & 0 & $3.83 \pm 0.05$ & $2.39 \pm 0.09$ & $37.5 \pm 1.6$ & $68.1 \pm 2.1$ \\
\hline & 20 & $4.12 \pm 0.10$ & $2.98 \pm 0.13$ & $27.7 \pm 1.8$ & $54.0 \pm 2.7$ \\
\hline & 47 & $4.46 \pm 0.12$ & $3.46 \pm 0.11$ & $22.6 \pm 0.5$ & $45.7 \pm 0.9$ \\
\hline & $60-69$ & $4.48 \pm 0.04$ & $3.57 \pm 0.06$ & $20.4 \pm 0.9$ & $41.7 \pm 1.5$ \\
\hline \multicolumn{2}{|c|}{$\begin{array}{l}\text { Simple main effect for } \\
\text { measurement time }\end{array}$} & $\begin{array}{c}F(3,12)=25.761, p< \\
0.0005, \text { partial } \eta^{2}= \\
0.866\end{array}$ & $\begin{array}{c}F(3,12)=37.638, p< \\
0.0005, \text { partial } \eta^{2}= \\
0.904\end{array}$ & $\begin{array}{c}F(3,12)=35.975, p< \\
0.0005, \text { partial } \eta^{2}= \\
0.900\end{array}$ & $\begin{array}{c}F(3,12)=36.057, p< \\
0.0005, \text { partial } \eta^{2}= \\
0.900\end{array}$ \\
\hline \multirow{4}{*}{ VPlu } & 0 & $3.71 \pm 0.07$ & $2.43 \pm 0.07$ & $34.5 \pm 1.7$ & $64.1 \pm 2.4$ \\
\hline & 20 & $4.00 \pm 0.06$ & $2.77 \pm 0.11$ & $30.8 \pm 20$ & $58.7 \pm 3.0$ \\
\hline & 47 & $4.33 \pm 0.09$ & $3.26 \pm 0.05$ & $24.7 \pm 1.1$ & $49.1 \pm 1.8$ \\
\hline & $60-69$ & $4.39 \pm 0.14$ & $3.21 \pm 0.15$ & $26.9 \pm 1.5$ & $52.6 \pm 2.4$ \\
\hline \multicolumn{2}{|c|}{$\begin{array}{l}\text { Simple main effect for } \\
\text { measurement time }\end{array}$} & $\begin{array}{c}F(3,15)=9.760, p< \\
0.005, \text { partial } \eta^{2}= \\
0.661\end{array}$ & $\begin{array}{c}F(3,15)=18.526, p< \\
0.0005, \text { partial } \eta^{2}= \\
0.787\end{array}$ & $\begin{array}{c}F(3,15)=13.968, p< \\
0.0005, \text { partial } \eta^{2}= \\
0.736\end{array}$ & $\begin{array}{c}F(3,15)=13.707, p< \\
0.0005, \text { partial } \eta^{2}= \\
0.733\end{array}$ \\
\hline \multicolumn{2}{|c|}{$\begin{array}{l}\text { Two-way interaction between } \\
\text { immunization and the time on } \\
\text { echocardiographic parameter }\end{array}$} & $\begin{array}{c}F(6,36)=3.755, p< \\
0.005, \text { partial } \eta^{2}= \\
0.385\end{array}$ & $\begin{array}{c}F(6,36)=8.669, p< \\
0.0005, \text { partial } \eta^{2}= \\
0.591\end{array}$ & $\begin{array}{c}F(6,36)=9.225, p< \\
0.0005, \text { partial } \eta^{2}= \\
0.606\end{array}$ & $\begin{array}{c}F(6,36)=9.460, p< \\
0.0005, \text { partial } \eta^{2}= \\
0.612\end{array}$ \\
\hline
\end{tabular}

\section{Results}

3.1. Immunization with VP1u Causes Progressive Worsening of Systolic Function in BALB/c Mice. We have followed heart morphology and cardiac function in mice by echocardiography over the course of 69 days after immunization (pi). Given timeframe encloses acute myocarditis (21 days pi) and DCM (60 days pi) phases reported for the established myosininduced myocarditis/DCM model $[17,18]$.

There was a statistically significant effect of the antigen on echocardiographic parameters in time-dependent manner. Immunizations with cardiac myosin and VP1u, but not with PBS, lead to statistically significant changes in LV enddiastolic and end-systolic diameters, fractional shortening, and LV ejection fraction over time (Table 1). Immunization with cardiac myosin and VPlu caused progressive dilatation of LV, resulting in gradual increase of LV diameter at systole and diastole (Figure 1). In good agreement with DCM phenotype, remodeling of LV was accompanied by the worsening of systolic function, measured by fractional shortening and ejection fraction. Immunization with cardiac myosin and VP1u resulted in statistically significant decrease of EF and FS as early as 20 days pi (Figure 1).

3.2. Immunization with VP1u Leads to Cardiac Fibrosis. 69 days after immunization hearts and lungs were examined histologically. Cardiac sections were stained with picrosirius red to visualize fibrosis and imaged under the white and polarized light (Figure 2). In cardiac sections of PBSimmunized mice no to mild fibrosis could be observed, evenly distributed through myocardium, physiologically more intensive around the vessels. Under polarized light, mostly green birefringence was observed, typical for thin collagen fibers. Myosin-immunized mice developed severe fibrosis clustering into multiple foci, mostly located in the pericardium, consisting of thick (red) and thin (green) collagen fibers. In VPlu animals, fibrotic foci developed around the vessels, mimicking pathological picture of "hypertensive" cardiomyopathy. Moreover, under the polarized light, extensive spread of interstitial fibrosis could be seen. The extent of cardiac fibrosis was graded by an experienced pathologist in a blinded manner. A Kruskal-Wallis $H$ test confirmed significantly different median fibrosis scores between studied groups, $H(2)=9.987, p=0.007$. Pairwise comparisons were performed using Dunn's procedure with a Bonferroni correction for multiple comparisons. Adjusted $p$ values are presented. This post hoc analysis revealed statistically significant differences in median fibrosis scores between the PBS (4.62) and cardiac myosin (13) $(p=0.013)$ and VPlu- (6.08) and cardiac myosin-immunized animals $(p=$ 0.028), but not between VPlu- (5.50) and PBS-immunized animals. 

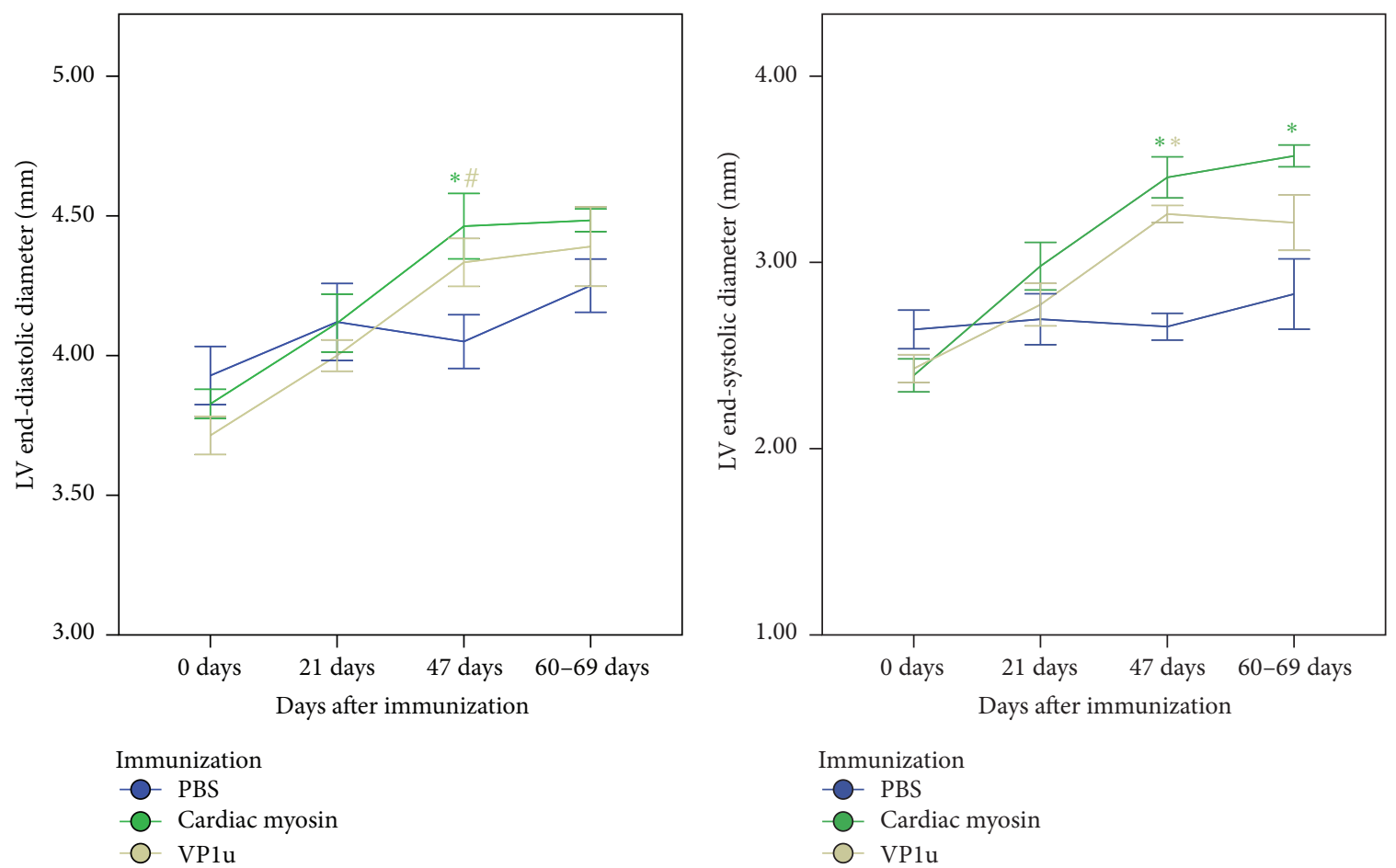

(a)
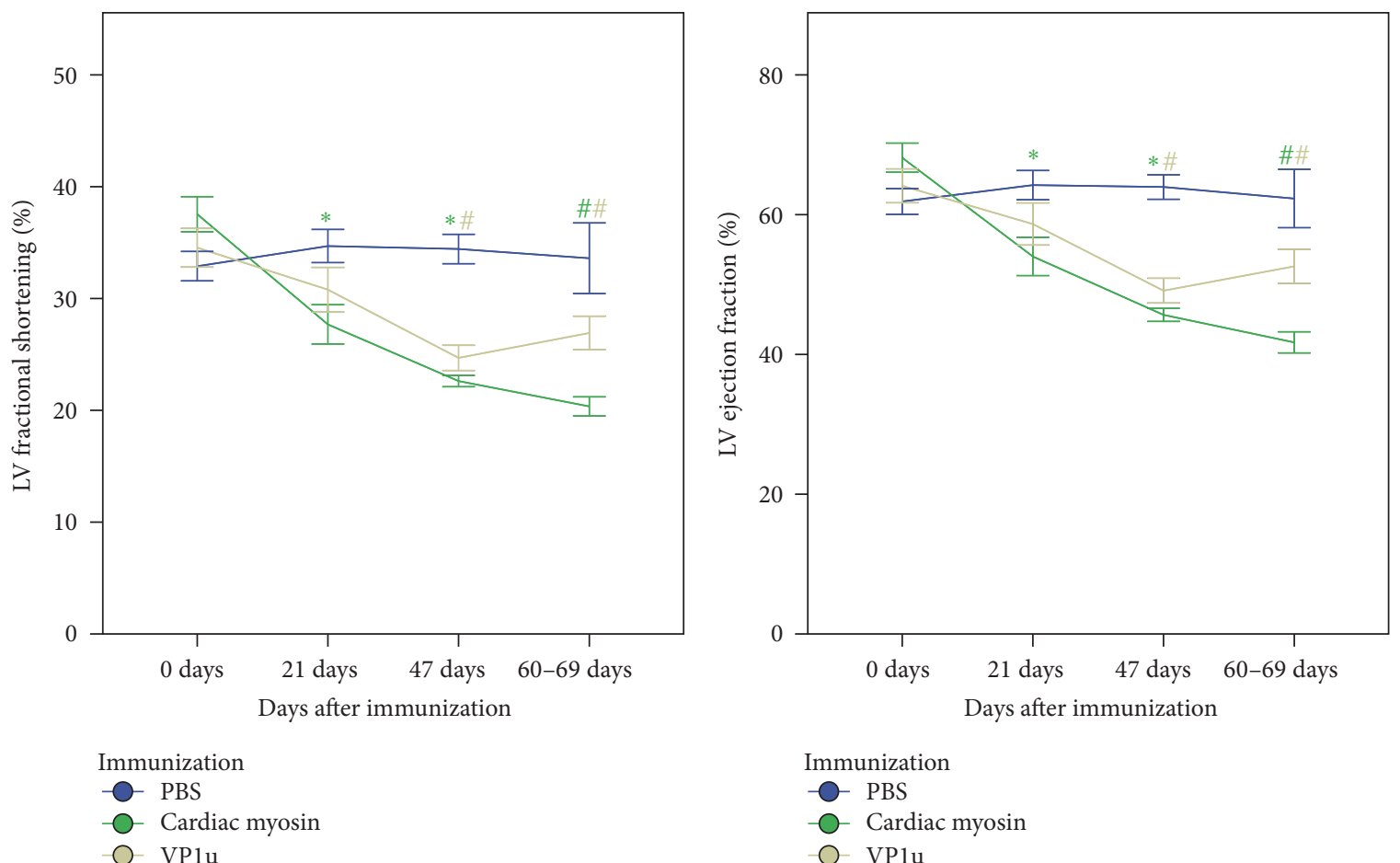
Immunization
- PBS
- Cardiac myosin
- VP1u

(c)

(d)

FIGURE 1: Serial echocardiographic measurements of immunized animals. All parameters were determined from serial echocardiography images obtained at given intervals in isoflurane-anesthetized mice. LV diameters were measured in M-mode at the short parasternal axis. Data are expressed as means \pm SEM; $n=6-10$; $*$ denotes $p<0.005$; \# denotes $p<0.05$, from 0 days. 


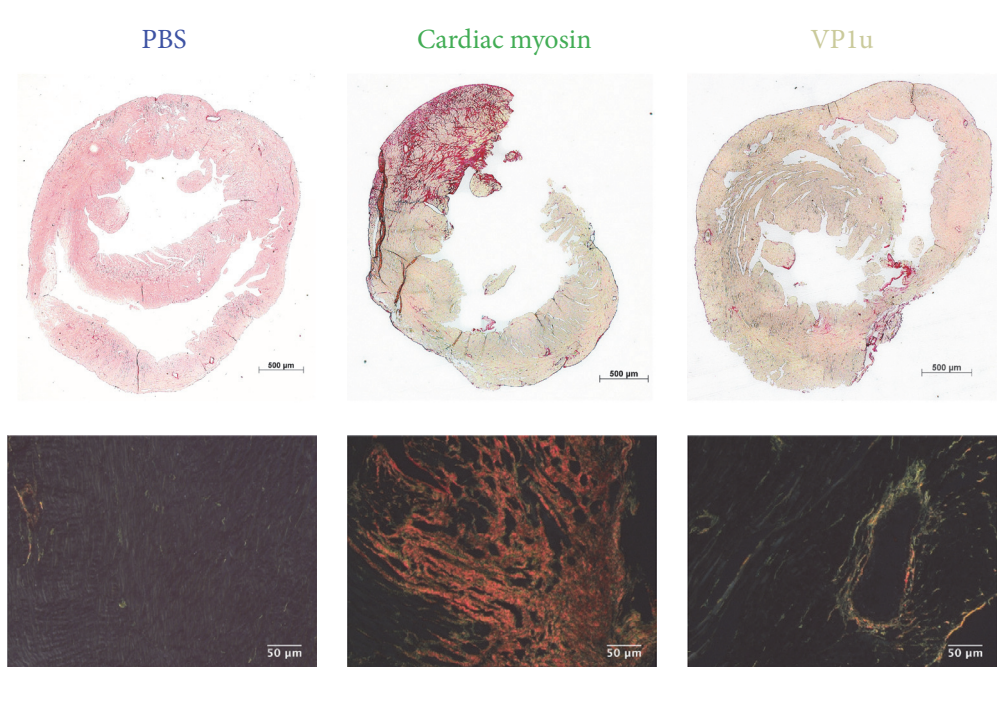

(a)

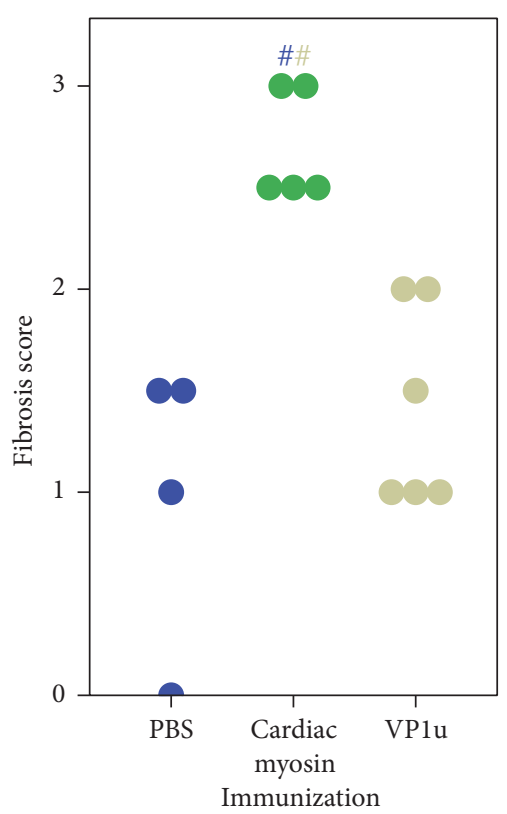

(b)

Figure 2: Fibrotic changes in the myocardium. Representative fields for each group are shown. Sections are photographed under white light (fibrosis-red, cardiac tissue-yellow to pink, upper panel) and under polarized light (thin collagen fibers are green, thick-red; the background is black, lower column). Distribution of fibrosis scores is given in (b). \# denotes $p<0.05$; color marks the compared group, $n=4-6$.

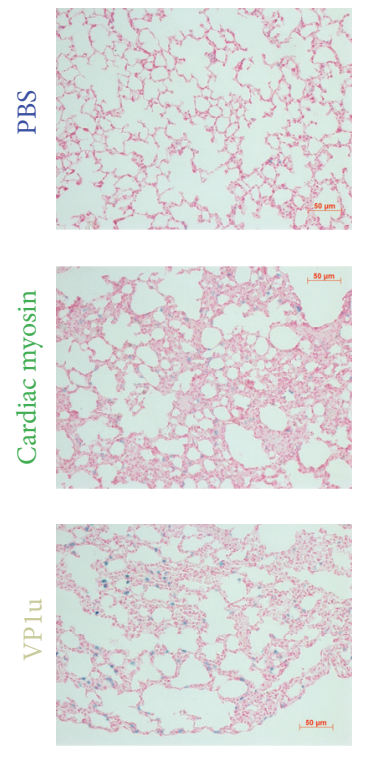

(a)

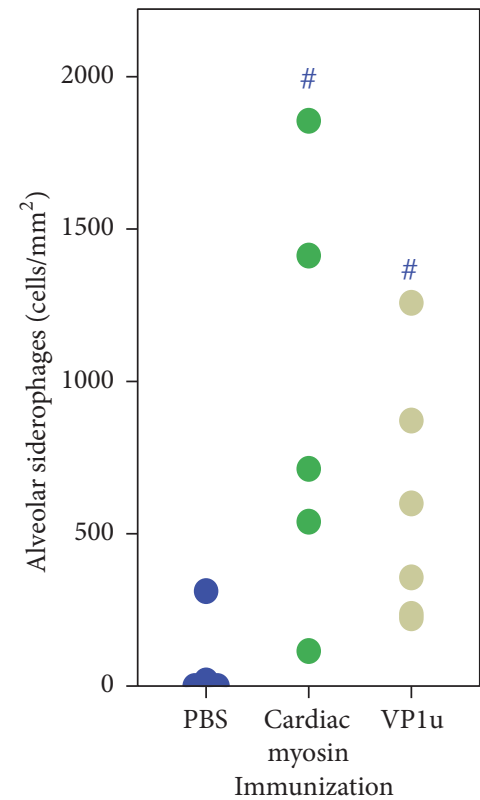

(b)

FIGURE 3: Accumulation of alveolar siderophages, indicative of heart failure in lungs of cardiac myosin- or VPlu-immunized animals. Representative fields are shown for each group. Siderophages stain blue in red counterstained lung tissue. Distribution of median alveolar siderophage densities is given in (b); \# denotes $p<0.05$; color marks the compared group, $n=4-6$.

3.3. Accumulation of Heart Failure Cells in Lungs of VP1uImmunized Animals. We have stained lungs of animals with Prussian blue for the visualization of siderophages known as the heart failure cells (Figure 3). Upon left heart failure, increased pulmonary blood pressure causes red blood cells to leak out, where they are engulfed by macrophages. The resulting accumulation of hemosiderin in macrophages stains blue. Cardiac myosin-immunized animals displayed the accumulation of alveolar siderophages compared to physiological levels observed in lungs of PBS-immunized 
TABLE 2: Top 10 dysregulated transcripts.

\begin{tabular}{|c|c|c|c|c|c|c|c|}
\hline \multicolumn{6}{|c|}{ Fold change $p$ value } & Fold change & $p$ value \\
\hline \multicolumn{8}{|c|}{ PBS versus cardiac myosin } \\
\hline Igkc & $\begin{array}{l}\text { Immunoglobulin kappa } \\
\text { constant }\end{array}$ & -2.47 & 0.04 & Timp4 & $\begin{array}{l}\text { Tissue inhibitor of } \\
\text { metalloproteinase } 4\end{array}$ & 2.94 & 0.01 \\
\hline Tfrc & Transferrin receptor & -1.80 & 0.01 & $\mathrm{Pdk} 4$ & $\begin{array}{l}\text { Pyruvate dehydrogenase } \\
\text { kinase, isoenzyme }\end{array}$ & 2.65 & 0.02 \\
\hline Tcrg-C4 & $\begin{array}{l}\text { T cell receptor gamma, } \\
\text { constant } 4\end{array}$ & -1.68 & 0.04 & Fkbp5 & FK506 binding protein 5 & 2.49 & 0.02 \\
\hline Mmp3 & Matrix metallopeptidase 3 & -1.45 & 0.05 & Zbtb16 & $\begin{array}{l}\text { Zinc finger and BTB domain } \\
\text { containing } 16\end{array}$ & 2.09 & 0.02 \\
\hline Comp & $\begin{array}{c}\text { Cartilage oligomeric matrix } \\
\text { protein }\end{array}$ & -1.36 & 0.05 & Fam107a & $\begin{array}{l}\text { Family with sequence } \\
\text { similarity } 107 \text {, mem }\end{array}$ & 2.06 & 0.02 \\
\hline Rrad & $\begin{array}{c}\text { Ras-related associated with } \\
\text { diabetes }\end{array}$ & -1.33 & 0.02 & Map3k6 & $\begin{array}{l}\text { Mitogen-activated protein } \\
\text { kinase kinase }\end{array}$ & 2.04 & 0.02 \\
\hline Ighm & $\begin{array}{l}\text { Immunoglobulin heavy } \\
\text { constant mu }\end{array}$ & -1.31 & 0.02 & Ucp3 & $\begin{array}{l}\text { Uncoupling protein } 3 \\
\text { (mitochondrial, pro }\end{array}$ & 1.97 & 0.03 \\
\hline Gpr22 & G protein-coupled receptor 22 & -1.06 & 0.01 & Cdknla & $\begin{array}{l}\text { Cyclin-dependent kinase } \\
\text { inhibitor } 1 \mathrm{~A}(\mathrm{p} 21)\end{array}$ & 1.73 & 0.05 \\
\hline Map2k6 & $\begin{array}{l}\text { Mitogen-activated protein } \\
\text { kinase kinase }\end{array}$ & -1.04 & 0.03 & Ms4a4a & $\begin{array}{l}\text { Membrane-spanning } \\
\text { 4-domain, subfamily A }\end{array}$ & 1.51 & 0.02 \\
\hline Igkj5 & $\begin{array}{l}\text { Immunoglobulin kappa } \\
\text { joining } 5\end{array}$ & -1.04 & 0.04 & Klhl38 & Kelch-like 38 & 1.45 & 0.04 \\
\hline \multicolumn{8}{|c|}{ PBS versus VPlu } \\
\hline Rrad & $\begin{array}{c}\text { Ras-related associated with } \\
\text { diabetes }\end{array}$ & -1.69 & 0.01 & Fkbp5 & FK506 binding protein 5 & 2.39 & 0.02 \\
\hline Kcnj2 & $\begin{array}{c}\text { Potassium inwardly-rectifying } \\
\text { channel }\end{array}$ & -1.05 & 0.01 & Timp4 & $\begin{array}{l}\text { Tissue inhibitor of } \\
\text { metalloproteinase } 4\end{array}$ & 2.04 & 0.03 \\
\hline Arhgap18 & $\begin{array}{c}\text { Rho GTPase activating } \\
\text { protein } 18\end{array}$ & -1.01 & 0.01 & Cdknla & $\begin{array}{l}\text { Cyclin-dependent kinase } \\
\text { inhibitor } 1 \mathrm{~A}(\mathrm{p} 21)\end{array}$ & 2.02 & 0.03 \\
\hline Ighm & $\begin{array}{l}\text { Immunoglobulin heavy } \\
\text { constant mu }\end{array}$ & -0.88 & 0.05 & Map3k6 & $\begin{array}{l}\text { Mitogen-activated protein } \\
\text { kinase kinase }\end{array}$ & 2.01 & 0.02 \\
\hline Lphn2 & Latrophilin 2 & -0.86 & 0.03 & Fam107a & $\begin{array}{l}\text { Family with sequence } \\
\text { similarity } 107, \text { mem }\end{array}$ & 2.00 & 0.03 \\
\hline Tfrc & Transferrin receptor & -0.82 & 0.02 & Serpinel & $\begin{array}{l}\text { Serine (or cysteine) peptidase } \\
\text { inhibitor }\end{array}$ & 1.72 & 0.01 \\
\hline Dusp18 & $\begin{array}{l}\text { Dual specificity phosphatase } \\
18\end{array}$ & -0.71 & 0.03 & Zbtb16 & $\begin{array}{l}\text { Zinc finger and BTB domain } \\
\text { containing } 16\end{array}$ & 1.71 & 0.03 \\
\hline Slc26a10 & $\begin{array}{l}\text { Solute carrier family } 26, \\
\text { member } 10\end{array}$ & -0.68 & 0.01 & 8430408G22Rik & $\begin{array}{c}\text { RIKEN cDNA } 8430408 G 22 \\
\text { gene }\end{array}$ & 1.69 & 0.02 \\
\hline Slc6a6 & $\begin{array}{c}\text { Solute carrier family } 6 \\
\text { (neurotransmitter transporter, } \\
\text { taurine), member } 6\end{array}$ & -0.68 & 0.04 & Ms4a4a & $\begin{array}{l}\text { Membrane-spanning } \\
\text { 4-domain, subfamily A }\end{array}$ & 1.60 & 0.02 \\
\hline Kctd12b & $\begin{array}{l}\text { Potassium channel } \\
\text { tetramerisation domain }\end{array}$ & -0.66 & 0.03 & Ctgf & $\begin{array}{l}\text { Connective tissue growth } \\
\text { factor }\end{array}$ & 1.32 & 0.01 \\
\hline \multicolumn{8}{|c|}{ Cardiac myosin versus VPlu } \\
\hline Pdk4 & $\begin{array}{l}\text { Pyruvate dehydrogenase } \\
\text { kinase, isoenzyme }\end{array}$ & -1.86 & 0.04 & Igkc & $\begin{array}{l}\text { Immunoglobulin kappa } \\
\text { constant }\end{array}$ & 2.54 & 0.03 \\
\hline Cesld & Carboxylesterase 1D & -1.27 & 0.03 & Trbj1-1 & T cell receptor beta joining 1-1 & 1.73 & 0.04 \\
\hline Apln & Apelin & -0.96 & 0.05 & Igj & $\begin{array}{l}\text { Immunoglobulin joining } \\
\text { chain }\end{array}$ & 1.56 & 0.04 \\
\hline Ttn & Titin & -0.96 & 0.04 & Comp & $\begin{array}{l}\text { Cartilage oligomeric matrix } \\
\text { protein }\end{array}$ & 1.38 & 0.04 \\
\hline Klf15 & Kruppel-like factor 15 & -0.93 & 0.04 & Mmp3 & Matrix metallopeptidase 3 & 1.34 & 0.05 \\
\hline Ctnnall & $\begin{array}{c}\text { Catenin (cadherin associated } \\
\text { protein), a }\end{array}$ & -0.93 & 0.01 & Nox4 & NADPH oxidase 4 & 1.07 & 0.04 \\
\hline
\end{tabular}


TABLE 2: Continued.

\begin{tabular}{|c|c|c|c|c|c|c|c|}
\hline & & Fold change & $p$ value & & & Fold change & $p$ value \\
\hline Tacc2 & $\begin{array}{c}\text { Transforming Acidic } \\
\text { Coiled-Coil Containing } \\
\text { Protein } 2\end{array}$ & -0.84 & 0.02 & Mcpt4 & Mast cell protease 4 & 1.03 & 0.04 \\
\hline $\mathrm{Scn} 4 \mathrm{a}$ & $\begin{array}{c}\text { Sodium channel, } \\
\text { voltage-gated, type IV }\end{array}$ & -0.82 & 0.03 & Tfre & Transferrin receptor & 0.98 & 0.01 \\
\hline Gpam & $\begin{array}{l}\text { Glycerol-3-phosphate } \\
\text { acyltransferase, mi }\end{array}$ & -0.74 & 0.03 & Rab2b & $\begin{array}{l}\text { RAB2B, member RAS } \\
\text { oncogene family }\end{array}$ & 0.94 & 0.04 \\
\hline Cited2 & $\begin{array}{l}\text { Cbp/p300-interacting } \\
\text { transactivator, wit }\end{array}$ & -0.73 & 0.04 & Snord71 & $\begin{array}{c}\text { Small nucleolar RNA, C/D } \\
\text { box } 71\end{array}$ & 0.93 & 0.04 \\
\hline Fbxo32 & F-box protein 32 & -0.73145 & 0.04 & Mgl2 & $\begin{array}{l}\text { Macrophage galactose } \\
\mathrm{N} \text {-acetyl-galactosamine } \\
\text { specific lectin } 2\end{array}$ & 0.91 & 0.01 \\
\hline Glul & $\begin{array}{l}\text { Glutamate-ammonia ligase } \\
\text { (glutamine synthase) }\end{array}$ & -0.68506 & 0.01 & Glipr1 & $\begin{array}{l}\text { GLI pathogenesis-related } 1 \\
\text { (glioma) }\end{array}$ & 0.84 & 0.04 \\
\hline
\end{tabular}

animals. A Kruskal-Wallis $H$ test confirmed significantly different median siderophage densities between studied groups, $H(2)=6.514, p=0.039$. Pairwise comparisons were performed using Dunn's procedure with a Bonferroni correction for multiple comparisons. Adjusted $p$ values are presented. This post hoc analysis revealed statistically significant differences in median fibrosis scores between the PBS (3.25) and cardiac myosin (10.6) $(p=0.043)$ and between VP1u- (9.0) and PBS-immunized animals $(p=0.049)$.

3.4. Transcriptomic Profiling of Immunized Animals. To gain molecular insights into VPlu immunization triggered heart damage we have performed gene expression profiling on left ventricular samples. 362 genes were found statistically significantly dysregulated between studied groups (Supplementary Table 1 in Supplementary Material available online at http://dx.doi.org/10.1155/2016/1627184). The list of top 10 dysregulated genes (Table 2) demonstrated shared and unique molecular features between myosin and VPlu triggered cardiac damage. In both animal models (compared to PBS control) matrix metallopeptidase 3 was upregulated, and tissue inhibitor of metalloprotease 4 was downregulated. This expression pattern resembles changes in the proteome, marking cardiac remodeling typical for the DCM [19, 20]. Moreover, commonly upregulated set of inflammationrelated genes (Igkc, Ighm, and Tcrg-C4) imply activation of the immune system in the myocardium. However, studied models share some differences in gene expression patterns. Immunization with the VPlu results in less pronounced immune activation of the myocardium, when compared to myosin-immunized mice, resulting in downregulation of immunity-related genes (Igkc, Igj, Trbj1-1, Mcpt4, and Mgl2). The cellular basis of this phenomenon could be related to a significant accumulation of CD45 positive immune cells in hearts of myosin-immunized animals (Figure 4). Set of genes involved in central energy metabolism ( $P d k 4$, Gpam, and Glul) was upregulated in VPlu-immunized animals. Speculatively, this could indicate a relatively spared energy metabolism in a less damaged myocardium of VPluimmunized animals; on another hand this might mean differing molecular mechanisms leading to heart damage.
Gene set enrichment analysis using KEGG annotation based gene set exemplifies differences between expression patterns of myosin- and VPlu-immunized animals. Gene sets related to inflammation and immune response are underrepresented in VPlu-immunized animals, whereas gene sets involved in energy metabolism are overrepresented.

\section{Discussion}

Echocardiographic and histological assessment of cardiac function and structure in VPlu-immunized animals revealed gradual remodeling of LV and worsening of systolic function. Observed changes are typical for DCM phenotype and follow the pattern of heart damage caused by established myosininduced myocarditis/DCM model, used in this work as a positive reference. Thus immunization with VP1u can be applied as a model to study clinically relevant B19V associated DCM.

Our findings are in good agreement with previous studies, demonstrating that active or passive immunization of susceptible mice strain with VPlu alone can elicit heart damage $[8,9]$. However, in this study we demonstrated that immunization with VPlu could progress beyond the phase of acute cardiac inflammation reported by Nie et al. and lead to LV dilatation, impaired systolic function, and cardiac fibrosis consistent with the development of DCM. This pattern closely resembles progression of heart damage elicited by the immunization with cardiac myosin derived peptide used in the established experimental autoimmune myocarditis/DCM model [10, 17].

However, cardiac damage in VPlu-immunized animals has unique features when compared to the cardiac myosinimmunized group. Animals in the VPlu-immunized group developed milder cardiac damage with less LV dilatation, lesser cardiac fibrosis, and fewer heart failure cells in lungs. Moreover, slight LV reverse remodeling was observed in VPlu-immunized animals 47 to 60-69 days after immunization. It is noteworthy that spontaneous recovery of cardiac function is well documented in clinical practice [21] and presented model could at least partly be used to study this relevant phenomenon. 


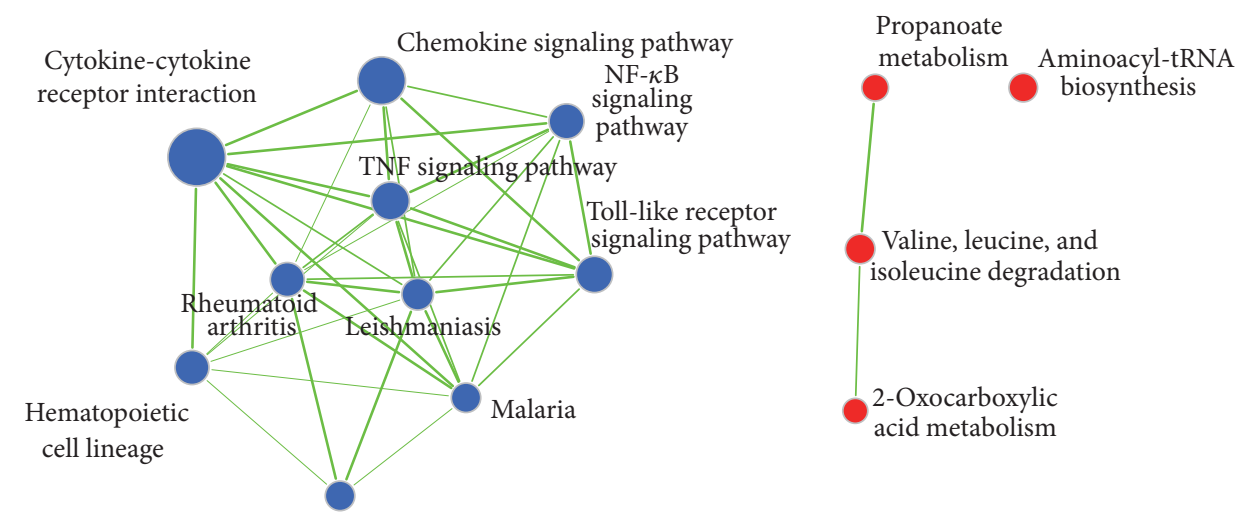

S. aureus infection

(a)
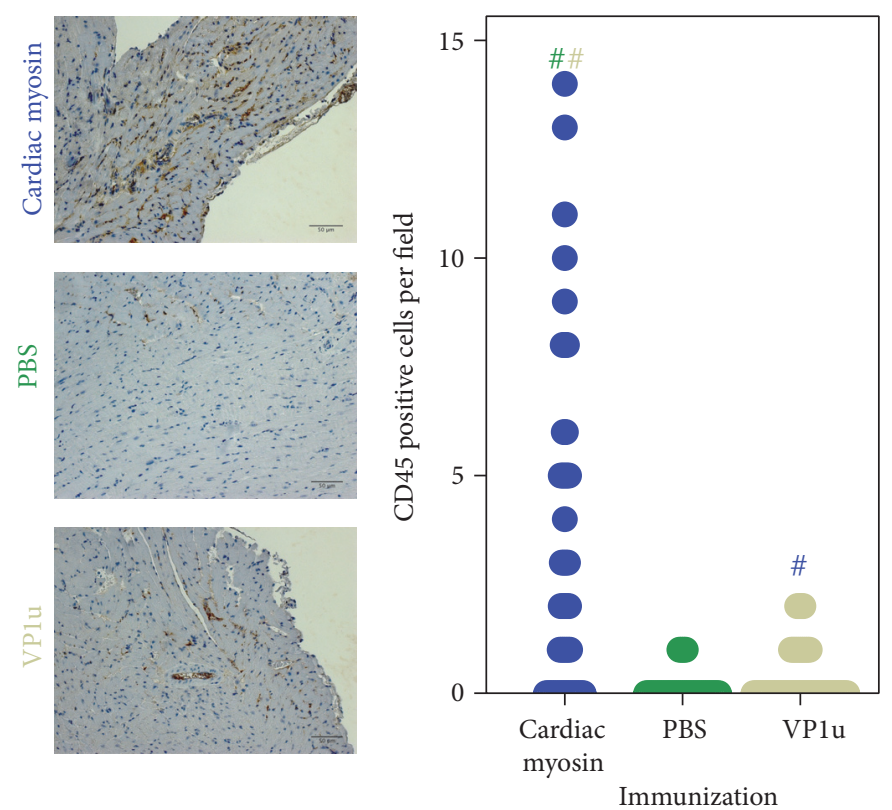

(b)

(c)

FIGURE 4: Immune infiltration in immunized animals. Network representation of under- and overrepresented gene sets between cardiac myosin- and VPlu-immunized animals (a). Gene sets related to immunity and infection are underrepresented in VPlu-immunized animals (blue nodes), whereas gene sets involved in catabolic and anabolic processes are overrepresented (red nodes). Accumulation of CD45 positive foci in cardiac myosin-immunized animals (b). Number of CD45 positive cells is given in (c); \# denotes $p<0.05$; color marks the compared group.

At the molecular level, comparative transcriptomic profiling revealed that immunization with VPlu resulted in less pronounced upregulation of inflammation-related genes than immunization with cardiac myosin. Conversely, immunization with VPlu upregulated set of energy metabolism genes. Speculatively, observed differences in gene expression patterns might reflect the varying severity of heart damage caused by the immunization with distinct antigens. Significant accumulation of CD45 positive immune cells in hearts of the cardiac myosin-immunized animals supports this observation. However, these differences might also imply differing molecular mechanisms leading to the heart damage.

Intriguingly, cardiac fibrosis in VPlu animals clustered around the vessels and resembled the histological picture of hypertrophic cardiomyopathy. It was shown that B19V myocarditis is an endothelial cell-mediated disease [22, 23] and anti-B19V antibodies alone can activate endothelial cells in vitro [24]. Thus, endothelium of cardiac vessels might be a primary autoimmune target in VPlu-immunized animals.

In conclusion, presented data indicate that immunization of $\mathrm{BALB} / \mathrm{c}$ mice with the recombinant VPlu protein causes heart damage compatible with the DCM phenotype. Described model can be useful to study clinically relevant B19V associated heart damage.

\section{Competing Interests}

The authors declare that they have no competing interests. 


\section{Acknowledgments}

This work has been funded by a Grant (MIP-011/2014) from the Research Council of Lithuania. The authors acknowledge the financial support of the Leducq Foundation (TNE-13CVD04) and the European Commission Marie Skłodowska-Curie Individual Fellowship (Titin Signals, 656636). The authors thank Roma Griniene for histochemical stainings performed. The authors are grateful to Microarray Technology core facility at Medical Research Centre and specially Carolina De La Torre and Carsten Sticht for microarray processing.

\section{References}

[1] C. Kawai, "From myocarditis to cardiomyopathy: mechanisms of inflammation and cell death: learning from the past for the future," Circulation, vol. 99, no. 8, pp. 1091-1100, 1999.

[2] S. Pankuweit and K. Klingel, "Viral myocarditis: from experimental models to molecular diagnosis in patients," Heart Failure Reviews, vol. 18, no. 6, pp. 683-702, 2013.

[3] E. D. Heegaard and K. E. Brown, "Human parvovirus B19," Clinical Microbiology Reviews, vol. 15, no. 3, pp. 485-505, 2002.

[4] E. Zuffi, E. Manaresi, G. Gallinella et al., "Identification of an immunodominant peptide in the parvovirus B19 VP1 unique region able to elicit a long-lasting immune response in humans," Viral Immunology, vol. 14, no. 2, pp. 151-158, 2001.

[5] T. Saikawa, S. Anderson, M. Momoeda, S. Kajigaya, and N. S. Young, "Neutralizing linear epitopes of b19 parvovirus cluster in the vP1 unique and vP1-vP2 junction regions," Journal of Virology, vol. 67, no. 6, pp. 3004-3009, 1993.

[6] C. Filippone, N. Zhi, S. Wong et al., "VPlu phospholipase activity is critical for infectivity of full-length parvovirus B19 genomic clones," Virology, vol. 374, no. 2, pp. 444-452, 2008.

[7] R. Leisi, N. Ruprecht, C. Kempf, and C. Ros, "Parvovirus B19 uptake is a highly selective process controlled by VPlu, a novel determinant of viral tropism," Journal of Virology, vol. 87, no. 24, pp. 13161-13167, 2013.

[8] X. Nie, G. Zhang, D. Xu et al., "The VP1-unique region of parvovirus B19 induces myocardial injury in mice," Scandinavian Journal of Infectious Diseases, vol. 42, no. 2, pp. 121-128, 2010.

[9] B.-S. Tzang, T.-M. Lin, C.-C. Tsai, J.-D. Hsu, L.-C. Yang, and T.C. Hsu, "Increased cardiac injury in NZB/W F1 mice received antibody against human parvovirus B19 VP1 unique region protein," Molecular Immunology, vol. 48, no. 12-13, pp. 15181524, 2011.

[10] D. Čiháková, R. B. Sharma, D. Fairweather, M. Afanasyeva, and N. R. Rose, "Animal models for autoimmune myocarditis and autoimmune thyroiditis," in Autoimmunity, vol. 102 of Methods in Molecular Medicine, pp. 175-193, Humana Press, Totowa, NJ, USA, 2004.

[11] J. Bogomolovas, B. Simon, M. Sattler, and G. Stier, "Screening of fusion partners for high yield expression and purification of bioactive viscotoxins," Protein Expression and Purification, vol. 64, no. 1, pp. 16-23, 2009.

[12] L. E. Teichholz, T. Kreulen, M. V. Herman, and R. Gorlin, "Problems in echocardiographic volume determinations: echocardiographic-angiographic correlations in the presence or absence of asynergy," The American Journal of Cardiology, vol. 37, no. 1, pp. 7-11, 1976.
[13] J. Schindelin, I. Arganda-Carreras, E. Frise et al., "Fiji: an opensource platform for biological-image analysis," Nature Methods, vol. 9, no. 7, pp. 676-682, 2012.

[14] A. Subramanian, H. Kuehn, J. Gould, P. Tamayo, and J. P. Mesirov, "GSEA-P: a desktop application for gene set enrichment analysis," Bioinformatics, vol. 23, no. 23, pp. 3251-3253, 2007.

[15] M. Kanehisa and S. Goto, "KEGG: kyoto encyclopedia of genes and genomes," Nucleic Acids Research, vol. 28, no. 1, pp. 27-30, 2000.

[16] D. Merico, R. Isserlin, O. Stueker, A. Emili, and G. D. Bader, "Enrichment map: a network-based method for gene-set enrichment visualization and interpretation," PLoS ONE, vol. 5, no. 11, Article ID e13984, 2010.

[17] M. Afanasyeva, D. Georgakopoulos, D. F. Belardi et al., "Quantitative analysis of myocardial inflammation by flow cytometry in murine autoimmune myocarditis: correlation with cardiac function," The American Journal of Pathology, vol. 164, no. 3, pp. 807-815, 2004.

[18] M. Afanasyeva, D. Georgakopoulos, and N. R. Rose, "Autoimmune myocarditis: cellular mediators of cardiac dysfunction," Autoimmunity Reviews, vol. 3, no. 7-8, pp. 476-486, 2004.

[19] C. V. Thomas, M. L. Coker, J. L. Zellner, J. R. Handy, A. J. Crumbley III, and F. G. Spinale, "Increased matrix metalloproteinase activity and selective upregulation in LV myocardium from patients with end-stage dilated cardiomyopathy," Circulation, vol. 97 , no. 17 , pp. 1708-1715, 1998.

[20] C. M. Tummalapalli, B. J. Heath, and S. C. Tyagi, "Tissue inhibitor of metalloproteinase-4 instigates apoptosis in transformed cardiac fibroblasts," Journal of Cellular Biochemistry, vol. 80, no. 4, pp. 512-521, 2001.

[21] A. D'Ambrosio, G. Patti, A. Manzoli et al., "The fate of acute myocarditis between spontaneous improvement and evolution to dilated cardiomyopathy: a review," Heart, vol. 85, no. 5, pp. 499-504, 2001.

[22] H. Mahrholdt, A. Wagner, C. C. Deluigi et al., "Presentation, patterns of myocardial damage, and clinical course of viral myocarditis," Circulation, vol. 114, no. 15, pp. 1581-1590, 2006.

[23] B. D. Bültmann, K. Klingel, K. Sotlar et al., "Fatal parvovirus b19-associated myocarditis clinically mimicking ischemic heart disease: an endothelial cell-mediated disease," Human Pathology, vol. 34, no. 1, pp. 92-95, 2003.

[24] B.-S. Tzang, C.-C. Tsai, C.-C. Chiu, J.-Y. Shi, and T.-C. Hsu, "Upregulation of adhesion molecule expression and induction of TNF- $\alpha$ on vascular endothelial cells by antibody against human parvovirus B19 VP1 unique region protein," Clinica Chimica Acta, vol. 395, no. 1-2, pp. 77-83, 2008. 


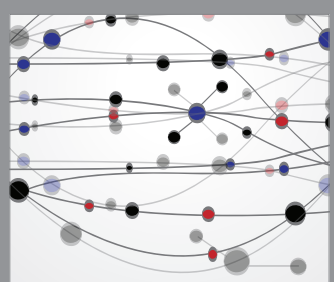

The Scientific World Journal
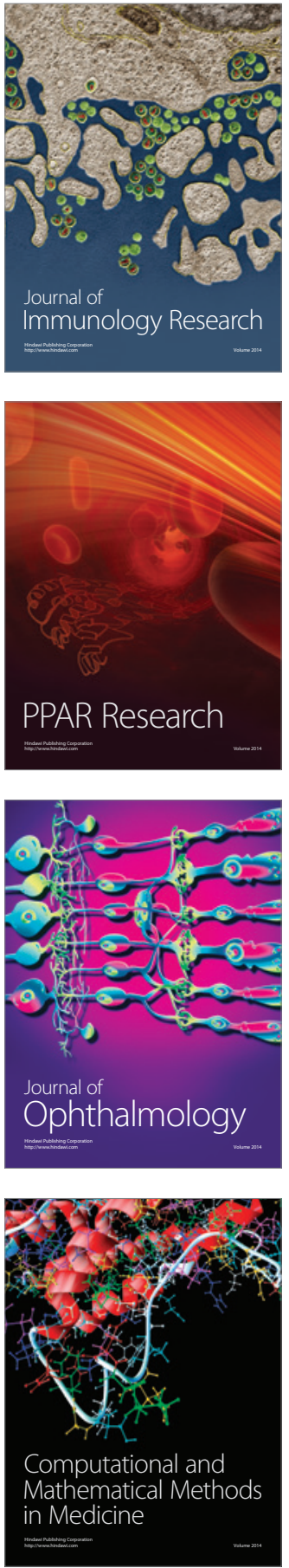

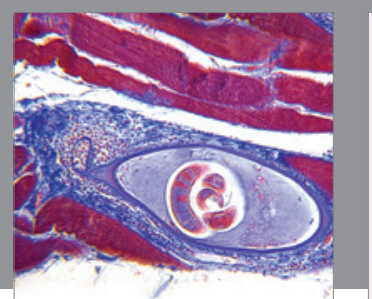

Gastroenterology Research and Practice

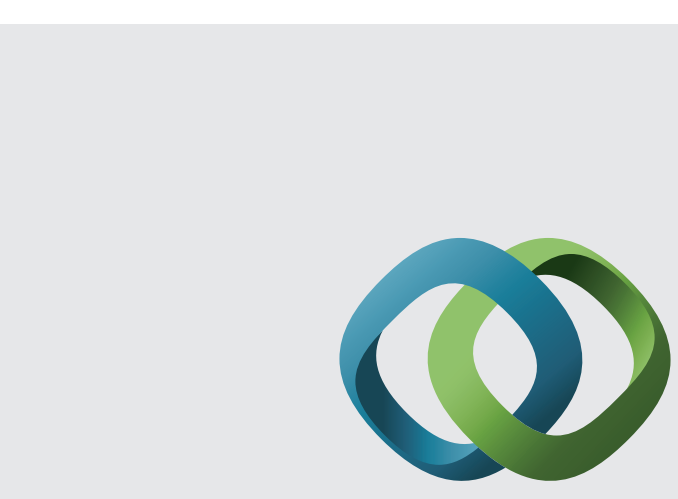

\section{Hindawi}

Submit your manuscripts at

http://www.hindawi.com
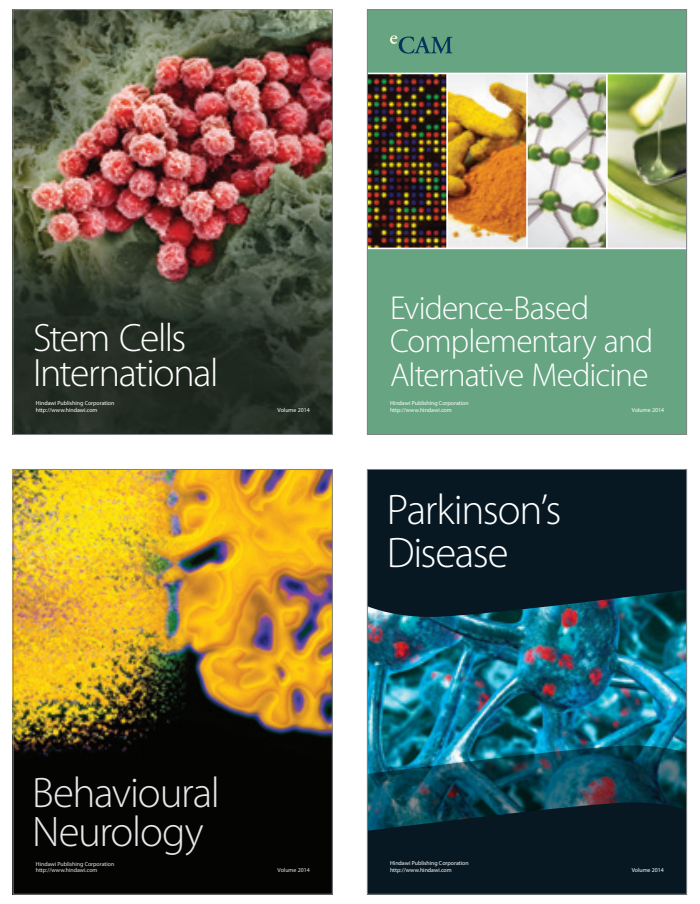
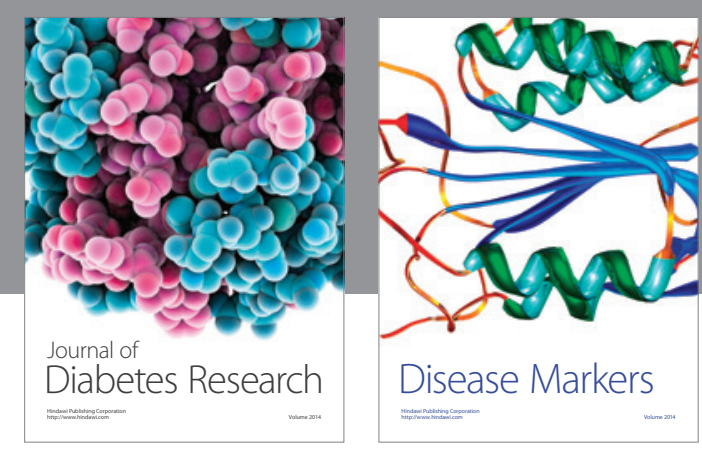

Disease Markers
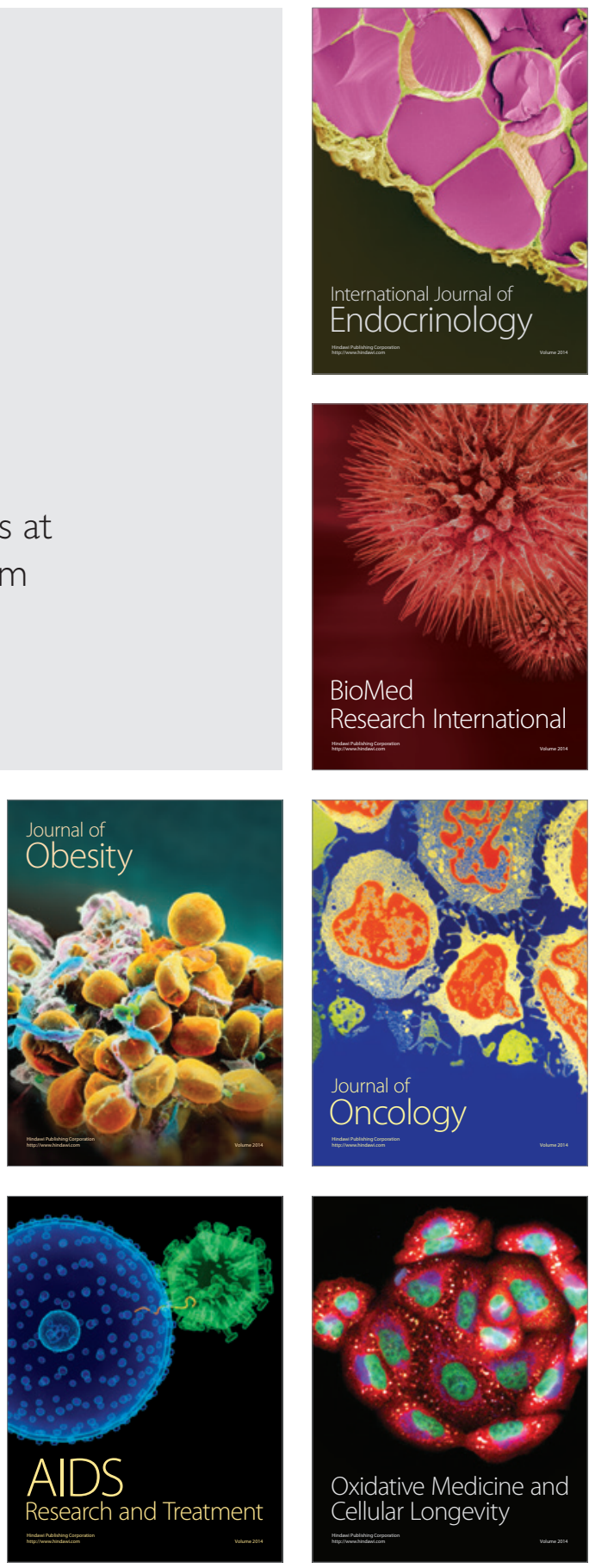